\title{
Erratum to: Linking Host Prokaryotic Physiology to Viral Lifestyle Dynamics in a Temperate Freshwater Lake (Lake Pavin, France)
}

\author{
S. Palesse - J. Colombet • A. S. Pradeep Ram • \\ T. Sime-Ngando
}

Published online: 23 December 2014

(C) Springer Science+Business Media New York 2014

\section{Erratum to: Microb Ecol (2014) \\ DOI 10.1007/s00248-014-0441-2}

The original version of the article unfortunately contains mistake on Tables 1 and 2 . The significant letter $\mathrm{b}$ for these tables under columns META and HYPO are missing. Please see below corrected version of Tables 1 and 2.

Table 1 Mean (range) environmental and microbial characteristics of epi-. meta- and hypolimnion of Lake Pavin. April - September 2011

\begin{tabular}{|c|c|c|c|}
\hline \multirow[t]{2}{*}{ Parameters } & \multicolumn{3}{|l|}{ Mean (Range) ${ }^{\mathrm{a}}$} \\
\hline & EPI & META & HYPO \\
\hline Water temperature $\left({ }^{\circ} \mathrm{C}\right)^{\mathrm{c}}$ & $15.3(9.2-19.6)$ & $8.4(4.8-14.8)$ & $4.2(4.1-4.3)$ \\
\hline Dissolved Oxygen $\left(\mathrm{mg} \mathrm{l}^{-1}\right)^{\mathrm{c}}$ & $9.6(8.4-11.4)$ & $12.7(8.7-15.8)$ & $5.0(3.6-6.8)$ \\
\hline Chlorophyll $a\left(\mu \mathrm{g}^{-1}\right)$ & $2.3(0.9-4.6)^{\mathrm{a}}$ & $7.0(1.4-12.9)^{\mathrm{a}, \mathrm{b}}$ & $1.5(0.4-2.7)^{b}$ \\
\hline Viral abundance $\left(10^{7} \mathrm{ml}^{-1}\right)$ & $2.8(0.8-4.4)^{\mathrm{a}}$ & $3.5(1.5-6.6)^{\mathrm{b}}$ & $0.6(0.2-1.1)^{\mathrm{a}, \mathrm{b}}$ \\
\hline Prokaryotic abundance $\left(10^{6}\right.$ cells ml $\left.{ }^{-1}\right)$ & $1.8(0.6-3.2)^{\mathrm{a}}$ & $2.3(0.7-4.0)^{b}$ & $0.7(0.4-1.0)^{\mathrm{a}, \mathrm{b}}$ \\
\hline LNA prokaryotic abundance $\left(10^{6}\right.$ cells $\left.\mathrm{ml}^{-1}\right)$ & $1.1(0.2-1.9)^{\mathrm{a}}$ & $1.2(0.4-2.6)^{b}$ & $0.4(0.2-0.5)^{\mathrm{a}, \mathrm{b}}$ \\
\hline HNA prokaryotic abundance $\left(10^{6}\right.$ cells $\left.\mathrm{ml}^{-1}\right)$ & $0.7(0.1-1.8)^{\mathrm{a}}$ & $1.0(0.3-2.3)^{b}$ & $0.3(0.1-0.6)^{\mathrm{a}, \mathrm{b}}$ \\
\hline Proportions of HNA cells (\%) & $39.7(11.2-61.3)$ & $44.0(22.1-69.8)$ & $42.4(22.1-57.6)$ \\
\hline Proportion of damaged prokaryotic cells (\%) & $32.8(21.0-49.1)$ & $37.3(19.4-57.0)$ & $38.0(21.1-57.1)$ \\
\hline Virus-to-prokaryote ratio & $16.8(7.5-27.1)^{\mathrm{a}}$ & $16.3(9.6-30.1)^{\mathrm{b}}$ & $8.9(4.4-12.7)^{\mathrm{a}, \mathrm{b}}$ \\
\hline Frequency of infected cells (\%) & $16.3(6.3-39.6)^{\mathrm{a}}$ & $21.5(5.8-36.6)^{b}$ & $10.3(2.4-34.8)^{\mathrm{a}, \mathrm{b}}$ \\
\hline Frequency of lysogenic cells (\%) & $1.9(0-11.1)^{\mathrm{a}}$ & $0.7(0-5.3)^{\mathrm{b}}$ & $0.2(0-1.4)^{\mathrm{a}, \mathrm{b}}$ \\
\hline Burst size (virus prokaryote ${ }^{-1}$ ) & $40(19-118)$ & $42(23-73)$ & $40(16-182)$ \\
\hline
\end{tabular}

Pairs of ${ }^{a}$ or of $^{b}$ indicate a significant difference between two of the three layers (Dunn's test. $p<0.05$ )

${ }^{\mathrm{c}}$ Significant differences between the three layers (Kruskal Wallis. $\mathrm{p}<0.05$ )

The online version of the original article can be found at http://dx.doi.org/ 10.1007/s00248-014-0441-2.

S. Palesse $\cdot$ J. Colombet $\cdot$ A. S. Pradeep Ram $(\bowtie) \cdot T$. Sime-Ngando Laboratoire Microorganismes: Génome et Environnement, UMR CNRS 6023 Clermont Université, Université Blaise Pascal, BP 80026, 63171 Aubière Cedex, France

e-mail: Pradeep_Ram.ANGIA_SRIRAM@univ-bpclermont.fr 
Table 2 Variances in environmental and microbiological parameters of epi-. meta- and hypolimnion of Lake Pavin. April - September 2011

\begin{tabular}{|c|c|c|c|c|c|}
\hline \multirow[t]{2}{*}{ Parameters } & \multicolumn{3}{|c|}{ Observed variance } & \multicolumn{2}{|l|}{$\mathrm{p}$-value } \\
\hline & EPI & META & HYPO & Bartlett's test & Fligner's test \\
\hline Water temperature & $8.65^{\mathrm{a}}$ & $2.68^{\mathrm{b}}$ & $0.01^{\mathrm{a}, \mathrm{b}}$ & $\mathrm{p}<0.0001$ & - \\
\hline Dissolved Oxygen & $0.64^{\mathrm{a}}$ & $4.69^{\mathrm{a}, \mathrm{b}}$ & $0.74^{\mathrm{b}}$ & $\mathrm{p}<0.0001$ & - \\
\hline Chlorophyll $a$ & $1.47^{\mathrm{a}}$ & $13.80^{\mathrm{a}, \mathrm{b}}$ & $0.60^{\mathrm{b}}$ & $\mathrm{p}<0.0001$ & - \\
\hline Viral abundance $\left(\times 10^{13}\right)$ & $3.95^{\mathrm{a}}$ & $13.18^{b}$ & $0.58^{\mathrm{a}, \mathrm{b}}$ & $\mathrm{p}<0.0001$ & - \\
\hline Prokaryotic abundance $\left(\mathrm{x} 10^{11}\right)$ & $5.07^{\mathrm{a}}$ & $7.40^{\mathrm{b}}$ & $0.40^{\mathrm{a}, \mathrm{b}}$ & - & $\mathrm{p}=0.0064$ \\
\hline HNA prokaryotic abundance $\left(\mathrm{x} 10^{11}\right)$ & $1.65^{\mathrm{a}}$ & $3.44^{\mathrm{b}}$ & $0.18^{\mathrm{a}, \mathrm{b}}$ & $\mathrm{p}<0.0001$ & - \\
\hline LNA prokaryotic abundance $\left(\times 10^{11}\right)$ & $2.44^{\mathrm{a}}$ & $2.25^{b}$ & $0.07^{\mathrm{a}, \mathrm{b}}$ & $\mathrm{p}<0.0001$ & - \\
\hline Proportion of damaged prokaryotic cells & 91.88 & 163.99 & 133.09 & ns & - \\
\hline Frequency of infected cells & 59.55 & 46.56 & 45.13 & - & ns \\
\hline Frequency of lysogenic cells ${ }^{c}$ & 7.60 & 1.61 & 0.07 & - & $\mathrm{p}=0.011$ \\
\hline
\end{tabular}

Pairs of ${ }^{\mathrm{a}}$ or of $^{\mathrm{b}}$ indicate a significant difference of variance between two of the three layers (Fisher-Snedecor. $\mathrm{p}<0.05$ )

$n s$ not significant

${ }^{c}$ Significant difference between each of the three layers 